\title{
Remarks on the Feynman Representation
}

\author{
By
}

\author{
Brian JEFFERIES*
}

\begin{abstract}
It is shown that there exists a complete space $\partial \mathrm{L}^{1}\left(M_{t}^{R}, M_{t}^{D}\right)$ of integrable functions such that for any potential $V$ with zero $H_{0}$-bound relative to the free Hamiltonian operator $H_{0}$ of a finite non-relativistic quantum system, the function $\exp \left[-\mathrm{i} \int_{0}^{t} V \circ X_{s} d s\right]$ belongs to $\partial \mathrm{L}^{1}\left(M_{t}^{K}, M_{t}^{D}\right)$, and the Feynman representation $\mathrm{e}^{-\mathrm{i}\left(H_{0}+V\right) t}=\int_{\Omega} \exp \left[-\mathrm{i} \int_{0}^{t} V \circ X_{s} d s\right] d M_{t}^{F}$ is valid.
\end{abstract}

\section{§ 0. Introduction}

Suppose that $\Delta$ is the selfadjoint extension on $\mathrm{L}^{2}\left(\mathbb{R}^{d}\right), d=1,2, \ldots$ of the Laplacian operator $\partial^{2} / \partial x_{1}^{2}+\ldots+\partial^{2} / \partial x_{d}^{2}$ acting on all smooth functions of compact support on $\boldsymbol{R}^{d}$. Then for the appropriate choice of the dimension $d$, the free Hamiltonian operator of a finite nonrelativistic quantum system is equivalent to the operator $H_{0}=-\frac{1}{2} \Delta$.

If $V: \boldsymbol{R}^{d} \rightarrow \boldsymbol{R}$ is a Borel measurable function representing the potential describing the interactions in the system, then under suitable conditions, $H=H_{0}+V$ is defined and selfadjoint on the domain of $H_{0}$, and it is equivalent to the Hamiltonian operator of the system.

The Feynman representation [2]

$$
\mathrm{e}^{-\mathrm{i} H t}=\int_{\Omega} \exp \left[-\mathrm{i} \int_{0}^{t} V \circ X_{s} d s\right] d M_{t}^{F}
$$

has recently been established for a large class of potentials $V$, including, for example, Coulomb interactions. For more singular $V$, such as the attractive $1 / r^{2}$ potential, the definite integrals seem to give a good description of the dynamics of the system [8]. A further

Communicated by H. Araki, June 17, 1985.

* Department of Mathematics, The Ohio State University, 231 West 18th Avenue, Columbus, Ohio 43210, U. S. A.

Present address; School of Mathematics and Physics, Macquavie University, North Ryde, 2113, Australia. 
extension has been considered for magnetic fields [3].

It was pointed out in [2] that the approach to integration with respect to the relevant operator valued set functions developed there is deficient in the sense that the space $\mathrm{L}^{1}\left(M_{t}^{K}, M_{t}^{D}\right)$ of (equivalence classes of) $M_{t}^{K}-M_{t}^{D}$-integrable functions is not complete in its natural topology. Examples of non-convergent Cauchy sequences in $\mathrm{L}^{1}\left(M_{t}^{K}, M_{t}^{D}\right)$ are provided by Johnson and Skoug [5] page 264.

The purpose of this note is to show that a slightly stronger integration process is sufficient to produce a complete space $\partial \mathrm{L}^{1}\left(M_{t}^{K}\right.$, $M_{t}^{D}$ ) of integrable functions, at the expense of diminishing the class $\mathscr{P}$ of potentials $V$ for which $\exp \left[-\mathrm{i} \int_{0}^{t} V \circ X_{s} d s\right]$ is integrable for each $t>0$. For example, the Feynman-Nelson approximating sequence will not converge in $\partial \mathrm{L}^{1}\left(M_{t}^{K}, M_{t}^{D}\right)$ for the attractive $1 / r^{2}$ potential [8].

However, the potentials with zero $H_{0}$-bound [6] page 190 belong to $\mathscr{P}$, so the Feynman representation (1) is still valid for the stronger integrals, and the convergence of the integral is closer to Feynman's original approach; namely, for each $t>0$, the operator $\int_{\Omega} \exp [-\mathrm{i}$. $\left.\int_{0}^{t} V \cdot X_{s} d s\right] d M_{t}^{F}$ belongs to the closure in the strong operator topology of the family $\left\{\int_{\Omega} s d M_{t}^{F}: s \in \operatorname{sim}\left(\mathscr{S}_{t}\right)\right\}$ of bounded linear operators on $\mathrm{L}^{2}\left(\mathbb{P}^{d}\right)$. In particular, $\mathscr{P}$ still contains Coulomb potentials.

In Section 1, slight modifications of convergence results for oneparameter semigroups of continuous linear operators on a Banach space [9], [10] are optained to cover the case of the uniform convergence of the limit of operators in the strong operator topology, as a certain parameter varies over compact sets.

The result is applied in Section 2 to the Feynman representation. An outline of integration with respect to closable set functions is given in the appendix.

\section{§1. A Uniform Product Formula}

The following lemma is probably well known, but because it is used repeatedly in what follows (and in [2] Lemma 4.3) it seems worthwhile to state and prove it explicitly.

Lemma $\mathbb{1}_{0}$ Let $E$ be a topological vector space. Let $\left\{T_{\tau}(a): \tau \in r\right.$, 
$a \in A\}$ be an equicontinuous family of linear operators on $E$ such that for each $x \in E, \lim _{\tau \in Y} T_{\tau}(a) x$ converges in $E$ uniformly for $a \in A$.

Then $\lim _{\tau \in Y} T_{\tau}(a) x$ converges in $E$ uniformly for $a \in A$ and as $x$ ranges over any precompact subset of $E$.

Proof. Let $K$ be a precompact subset of $E$. Let $U$ be a closed neighbourhood of zero in $E$. Then there exists a finite subset $\left\{x_{i}: i=1, \ldots, n\right\}$ of $K$ such that $K \subset \cup\left(x_{i}+V\right)$ and $V$ is a neighbourhood of zero in $E$ such that

$$
\underset{\substack{a \in A \\ \tau \in Y}}{\cup} T_{\tau}(a) V \subset U,
$$

by virtue of equicontinuity.

If the limit of $T_{\tau}(a), \tau \in \mathcal{Y}$ is denoted by $T(a)$ for each $a \in A$, then for $\tau \in \mathcal{Y}$ sufficiently large, $T_{\tau}(a) x_{i}-T(a) x_{i} \in U$ for $i=1, \ldots, n$ and every $a \in A$, by the uniform convergence.

Therefore, for any $x \in K$,

$$
\left[T_{\tau}(a)-T(a)\right] x \in U+U-U
$$

for every $a \in A$, giving the result.

The domain of an operator $T$ is denoted by $\mathscr{D}(T)$. The space of all continuous linear operators on a Banach space $X$ is written as $\mathscr{L}(X)$.

Lemma 2。 Let $X$ be a Banach space. Let $Y$ be a non-empty set and $B: \mathscr{D}(B) \rightarrow X$ a linear operator on $X$.

Suppose that there exist a number $M>0$ and linear operators $A(\tau)$ : $\mathscr{D}(A(\tau)) \rightarrow X, \tau \in \mathcal{Y}$ such that for each $\tau \in \mathcal{Y}$ the operator $A(\tau)+B$ defined on $\mathscr{D}(A(\tau)) \cap \mathscr{D}(B)$ is closable, and its closure $T(\tau)$ generates a $C_{0^{-}}$ semigroup $\mathrm{e}^{T(\tau) t}, t>0$ such that

$$
\left\|\mathrm{e}^{T(\tau) t}\right\| \leq M
$$

for every $0 \leq t \leq 1$.

Suppose also that for each $y \in Y=\bigcap_{\tau \in \Upsilon} \mathscr{D}(A(\tau)) \cap \mathscr{D}(B)$, the set $\{A(\tau) y: \tau \in \mathcal{Y}\}$ is a relatively compact subset of $X$, and $Y$ is dense in $X$.

Then for each $y \in Y$,

$$
\left(\mathrm{e}^{T(\tau) s}-I\right) y / s \rightarrow(A(\tau)+B) y
$$


as $s \downarrow 0$, uniformly for $\tau \in \Upsilon$.

Proof. For each $f \in Y, \tau \in \mathcal{Y}$

$$
\mathrm{e}^{T(\tau) t} f=f+\int_{0}^{t} \mathrm{e}^{T(\tau) s}(A(\tau)+B) f d s
$$

for each $t>0$. The integral is the limit of Riemann sums.

By virtue of the assumed uniform boundedness of $\mathrm{e}^{T(\tau) s}, \tau \in Y$ about $s=0$, it follows that $\mathrm{e}^{T(\tau) s} f \rightarrow f$ uniformly for $\tau \in Y$ as $s \rightarrow 0$, so $\mathrm{e}^{T(\tau) s} x \rightarrow x$ uniformly for $\tau \in \mathcal{Y}$ as $s \rightarrow 0$, for each $x \in X$ by equicontinuity. We have used the fact that $\{A(\tau) f: \tau \in \Upsilon\}$ is a bounded subset of $X$.

Moreover

$$
\left(\mathrm{e}^{T(\tau) t}-I\right) f / t-(A(\tau)+B) f=\int_{0}^{t}\left(\mathrm{e}^{T(\tau) s}-I\right)(A(\tau)+B) f d s / t,
$$

so the result follows from the precompactness of $\{A(\tau) f: \tau \in Y\}$ and Lemma 1.

The uniform product formula for contraction semigroups follows from a minor variation of Nelson's proof [8] of the Trotter product formula.

Theorem 1. Let $X$ be a Banach space. Let $B$ be a bounded operator on $X$. For each $\tau \in Y$, let $A(\tau)$ be the generator of a $C_{0}$-contraction semigroup such that the set $Y=\bigcap_{\tau \in \Gamma} \mathscr{D}(A(\tau))$ is a core for each $A(\tau)$, $\tau \in \mathcal{Y}$, and $\tau \mapsto A(\tau) y, \tau \in \mathcal{Y}$ is continuous for each $y \in Y$, with $Y$ metrizable and precompact. Then for each $t>0$

$$
\mathrm{e}^{(A(\tau)+B) t}=\lim _{n \rightarrow \infty}\left[\mathrm{e}^{B t / n} \mathrm{e}^{A(\tau) t / n}\right]^{n}
$$

in the strong operator topology on $\mathscr{L}(X)$, uniformly for $\tau \in \Upsilon$.

Proof. It can be assumed from the outset that $\left\|\mathrm{e}^{B t}\right\| \leq 1$ for all $t>0$, since $B$ can be replaced by $B-\|B\|_{\infty}$ if necessary.

Let $R^{\tau}(t)=\mathrm{e}^{(A(\tau)+B) t}, S^{\tau}(t)=\mathrm{e}^{A(\tau) t}, T(t)=\mathrm{e}^{B t}$ and $U^{\tau}(t)=T(t) S^{\tau}(t)$ for each $t>0$ and $\tau \in Y$. For $\tau \in Y$ and $s>0$, set $x_{s}^{\tau}=R^{\tau}(s) x$ for each $x \in X$. Then for $t>0$

$$
\begin{gathered}
\left\|R^{\tau}(t) x-\left[U^{\tau}(t / n)\right]^{n} x\right\|=\| \sum_{j=0}^{n-1}\left[U^{\tau}(t / n)\right]^{j}\left(R^{\tau}(t / n)-U^{\tau}(t / n)\right) \\
{\left[R^{\tau}(t / n)\right]^{n-j-1} x \|}
\end{gathered}
$$




$$
\leq \sup _{0 \leq s \leq t} n\left\|\left(R^{\tau}(t / n)-U^{\tau}(t / n)\right) x_{s}^{\tau}\right\|
$$

for each $x \in X$.

Let $x \in Y$. By Lemma 2

$$
\left(R^{\tau}(s)-I\right) x / s \rightarrow(A(\tau)+B) x
$$

as $s \downarrow 0$, uniformly for $\tau \in Y$.

The continuity of $R^{\tau}(\cdot)$ and $S^{\tau}(\cdot)$ is obviously uniform for $\tau \in \Upsilon$, because it is for a dense set of vectors, and the families of operators are equicontinuous. Now appealing to Lemma 1 ,

$$
\begin{aligned}
\left(U^{\tau}(s)-I\right) x / s & =T(s) A(\tau) x+T(s)\left[\left(S^{\tau}(s)-I\right) x / s-A(\tau) x\right] \\
& +(T(s)-I) x / s \rightarrow A(\tau) x+0+B x, \quad s \downarrow 0
\end{aligned}
$$

uniformly for $\tau \in \Upsilon$. Here we use the fact that $\{A(\tau) x: \tau \in \mathcal{Y}\}$ is a precompact subset of $X$, and so is $\left\{\left(S^{\tau}\left(s_{n}\right)-I\right) x / s_{n}: n=1,2, \ldots, \tau \in Y^{r}\right\}$ for $s_{n} \rightarrow 0$ as $n \rightarrow \infty$. The last assertion follows from strong resolvent convergence [9] by the continuity of $A(\cdot) y, y \in Y$, and an elementary topological argument, given explicitly, for example, in [4] Lemma 4.1. Therefore, $\lim _{n \rightarrow \infty} n\left[R^{\tau}(t / n)-U^{\tau}(t / n)\right] x=0$ in $X$, uniformly for $\tau \in \Upsilon$.

Let $Z$ be the space of continuous functions $\tau \mapsto z^{\tau}$, $\tau \in Y$ from $r$ into $X$ such that $z^{\tau} \in \mathscr{D}(A(\tau))$ for each $\tau \in \gamma$ and the function $\tau \mapsto A(\tau) z^{\tau}, \tau \in Y$ is continuous. Equip $Z$ with the norm

$$
\|z\|_{z}=\sup _{\tau \in Y}\left\|z^{\tau}\right\|+\sup _{\tau \in Y}\left\|A(\tau) z^{\tau}\right\|, \quad z \in Z .
$$

Then $Z$ is a Banach space because $A(\tau)$ is closed for each $\tau \in Y$.

The same argument as in Lemma 2 and as above shows that for each $z \in Z$

$$
\lim _{n \rightarrow \infty} n\left[R^{\tau}(t / n)-U^{\tau}(t / n)\right] z^{\tau}=0
$$

in $X$ uniformly for $\tau \in Y$. By the uniform boundedness principle, there exists $G>0$ such that

$$
\sup _{\tau \in \Gamma}\left\|n\left[R^{\tau}(t / n)-U^{\tau}(t / n)\right] z^{\tau}\right\| \leq C\|z\|_{Z}
$$

for all $n=1,2, \ldots$ and $z \in Z$. The convergence as $n \rightarrow \infty$ is uniform as $Z$ varies over compact subsets of $Z$.

Again, $A(\cdot) y, y \in Y$ is continuous, so $\tau \rightarrow R^{\tau}(s), \tau \in \gamma$ is continuous for each $s>0$ by strong resolvent convergence. The uniform continuity of $R^{\tau}(\cdot)$ for $\tau \in Y$ on $[0, t]$ shows that

$$
\left\{x_{s}^{:}: 0 \leq s \leq t\right\}
$$


is a compact subset of $Z$.

The right-hand side of (2) therefore goes to zero, uniformly for $\tau \in \mathcal{Y}$ and for $t$ in compact subsets of $[0, \infty[$.

The notion of strong resolvent convergence needs to be extended to uniform convergence as a parameter varies over a set. In this case, the argument of Kato [6] IX. 2.16 requires less modification than does Nelson's proof of the Trotter product formula.

Let $E_{a}, a \in A$ be dense subspaces of the topological vector space $E$. The collection $\left\{E_{a}: a \in A\right\}$ is said to be uniformly dense in $E$ if for each $x \in E$ there exists a directed set $Z$ and $x_{\zeta}^{a} \in E_{a}, a \in A, \zeta \in Z$ such that $\lim _{\zeta \in Z} x_{\zeta}^{a}=x$ in $E$ uniformly for $a \in A$.

The condition is clearly satisfied whenever $\underset{a \in A}{\cap E_{a}}$ is dense in $E$.

Theorem 2. Let $T(a), T_{n}(a), n=1,2, \ldots, a \in A$ be the generators of $C_{0}$-semigroups. Suppose that there exist $M, \beta>0$ such that

$$
\left\|\mathrm{e}^{T_{n}(a) t}\right\| \leq M \mathrm{e}^{\beta t}
$$

for all $t>0, a \in A, n=1,2, \ldots$

Suppose also that $A$ is precompact and metrizable, and the operator valued function $a \mapsto T(a), a \in A$ is continuous in the sense of strong resolvent convergence.

If $\{\mathscr{D}(T(a)): a \in A\}$ is uniformly dense in $E$, and for some $\lambda \in \mathbb{C}$ with $\operatorname{Re} \lambda>\beta$,

$$
\left(\lambda I-T_{n}(a)\right)^{-1} \rightarrow(\lambda I-T(a))^{-1}
$$

uniformly for $a \in A$, in the strong operator lopology as $n \rightarrow \infty$, then

$$
\mathrm{e}^{T_{n}(a) t} \rightarrow \mathrm{e}^{T(a) t}
$$

in the strong operator topology, uniformly for $a \in A$ and as $t$ ranges over any compact subset of $[0, \infty]$, as $n \rightarrow \infty$.

Conversely, if for each $t>0$,

$$
\mathrm{e}^{T_{n}(a) t} \rightarrow \mathrm{e}^{T(a) t}
$$

uniformly for $a \in A$, in the strong operator topology as $n \rightarrow \infty$, then

$$
\left(\lambda I-T_{n}(a)\right)^{-1} \rightarrow(\lambda I-T(a))^{-1}
$$

in the strong operator topolog $\gamma$, uniformly for $a \in A$, and for $\lambda$ in compact subsets of $\{z \in \mathbb{C}: \operatorname{Re} z>\beta\}$. 
Proof. The first statement follows by systematically applying Lemma 1 to the proof of [6] IX.2.16. The converse follows by noting that

$$
\begin{aligned}
\sup _{a \in A}\left\|\left[\left(\lambda I-T_{n}(a)\right)^{-1}-(\lambda I-T(a))^{-1}\right] x\right\| \leq \int_{0}^{\infty} \mathrm{e}^{-\alpha t} \sup _{a \in A} \|\left[\mathrm{e}^{T_{n}(a) t}\right. & \left.-\mathrm{e}^{T(a) t}\right] x \| d t
\end{aligned}
$$

for every $\lambda \in \mathbb{C}$ with $\operatorname{Re} \lambda=\alpha>\beta$, and every vector $x_{0}$ Dominated convergence gives the result.

\section{\$ 2. Boundary Integration}

The notation adopted in the appendix will be adhered to in this section. The lack of completeness of the space $\mathrm{L}^{1}\left(M_{t}^{K}, M_{t}^{D}\right)$ introduced in [2] is a consequence of the space $H_{c}(D)$ being incomplete; this is the space of continuous functions on $D$, analytic in the interior of $D$, and equipped with the topology of uniform convergence on compact subsets of the interior of $D$. In some ways, this is a natural space to use because we are dealing with a boundary-value problem for holomorphic functions-solutions are constructed in the interior of $D$ so that boundary-values are taken on continuously - a time-honoured technique in analysis.

One way to ensure that boundary-values are taken on continuously is to approximate by holomorphic functions which have this property, uniformly on compact subsets of $D$. It is to be expected that such solutions have better stability properties than those which are constructed by approximation in the interior of $D$.

Let $\mathscr{C}(D)$ denote the family of all compact subsets of $D$. For each $C \in \mathscr{C}(D)$ and $\phi \in \mathbb{L}^{2}\left(\mathrm{~F}^{d}\right)$, set $\Xi_{t}^{C, \phi}=\left\{\left(M_{t}^{z} \phi, \phi\right): z \in C,\|\phi\|_{2} \leq 1\right\}$ and $\Xi_{t}=\left\{\Xi_{t}^{C, \phi}:\|\phi\|_{2} \leq 1, C \in \mathscr{C}(D)\right\}$, for each $t>0$.

For each $t>0$, the increasing family of sub-semi-algebras is the usual one, $\left.\left.\mathscr{S}_{J}, J \subset\right] 0, t\right]$ finite. The set functions $\left(M_{t}^{z} \phi, \phi\right), \phi, \phi \in$ $\mathbb{L}^{2}\left(\mathbb{R}^{d}\right), z \in D$ are viewed as elements of ba $\left(\mathscr{S}_{J}, \mathbb{C}\right)$.

It is easily checked, as in [2] Theorem 3.4, that for each $t>0$, $\Xi_{t}$ is $\Gamma_{t}$-closable. Because the space ba $\left(\mathscr{S}_{J}, \widetilde{\cup}\right)$ is complete, every function (identifying a function with its equivalence class, as usual) belonging to the domain $\mathscr{D}\left(\bar{I}_{\Gamma_{t} \varepsilon_{t}}\right)$ of the closed linear map $\bar{I}_{\Gamma_{t} \Xi_{t}}$ is $\Gamma_{t^{-}} \Xi_{t}$-integrable. Although this is a simplification, the task of 
verifying that a function is $\Gamma_{t}-\Xi_{t}$-integrable is more difficult.

A $\Gamma_{t}-\Xi_{t}$-integrable function is said to be boundary- $M_{t}^{K}-M_{t}^{D}$-integrable, or briefly, $\partial-M_{t}^{K}-M_{t}^{D}$-integrable. The space of (equivalence classes of) $\partial-M_{t}^{K}-M_{t}^{D}$-integrable functions equipped with the coarsest topology for which both the inclusion of $\mathrm{L}^{1}\left(\Gamma_{t}, \bar{\Xi}_{t}\right)$ in $\mathrm{L}^{1}\left(M_{t}^{K}\right)$ and the map $\bar{I}_{\Gamma_{t} \Xi_{t}}$ are continuous is denoted by $\partial \mathrm{L}^{1}\left(M_{t}^{K}, M_{t}^{D}\right)$. Because the map $\bar{I}_{\Gamma_{t} \Xi_{t}}$ is closed, the space $\partial \mathrm{L}^{1}\left(M_{t}^{K}, M_{t}^{D}\right)$ is a complete locally convex Hausdorff topological vector space. The completeness of the space of $\partial-M_{t}^{K}-M_{t}^{D}$-integrable functions is obviously a desirable property.

The cardinality of the topology of $\partial \mathrm{L}^{1}\left(M_{t}^{K}, M_{t}^{D}\right)$ is the same as the cardinality of the continuum, because we are using the collection of all finite subsets of the interval $] 0, t]$; the topology may or may not be viewed as large, depending on whether or not one believes in the continuum hypothesis. In any case, $\partial \mathrm{L}^{1}\left(M_{t}^{K}, M_{t}^{D}\right)$ is surely not a Fréchet space.

Because $\partial \mathrm{L}^{1}\left(M_{t}^{K}, M_{t}^{D}\right) \subset \mathrm{L}^{1}\left(M_{t}^{K}, M_{t}^{D}\right)$, the integrals $f M_{t}^{z}: \mathscr{S}_{t} \rightarrow$ $\mathscr{L}\left(\mathrm{L}^{2}\left(\boldsymbol{R}^{d}\right)\right)$ of $\partial-M_{t}^{K}-M_{t}^{D}$-integrable functions are defined in exactly the same way as for $M_{t}^{K}-M_{t}^{D}$-integrable functions [2] Theorem 3.5.

Theorem 3. Let $V: \boldsymbol{R}^{d} \rightarrow \mathbb{R}$ be a Borel measurable function such that the domain of the operator of multiplication by $V$ contains $\mathscr{D}\left(H_{0}\right)$, and for each $a>0$, there exists $b>0$ such that

$$
\|V f\|_{2} \leq a\left\|H_{0} f\right\|_{2}+b\|f\|_{2}
$$

for every $f \in \mathscr{D}\left(H_{0}\right)$.

Then for each $t>0$, the function

$$
\exp \left[-\mathrm{i} \int_{0}^{t} V \circ X_{s} d s\right]: \omega \rightarrow \exp \left[-\mathrm{i} \int_{0}^{t} V \circ X_{s}(\omega) d s\right], \omega \in \Omega_{t}
$$

is defined on a set $\Omega_{t}$ of full $M_{t}^{K}$-measure and it is $\partial-M_{t}^{K}-M_{t}^{D}$-integrable.

Furthermore, $H_{0}+V$ is selfadjoint on $\mathscr{D}\left(H_{0}\right)$ and

$$
\mathrm{e}^{-\mathrm{i}\left(H_{0}+V\right) t}=\int_{\Omega} \exp \left[-\mathrm{i} \int_{0}^{t} V \circ X_{s} d s\right] d M_{t}^{F} .
$$

Proof. First suppose that $V$ is continuous and bounded. Define

$$
f_{n}(\omega)=\prod_{j=1}^{n} \exp [-\mathrm{i} V(\omega(j t / n)) t / n]
$$

for $\omega \in \Omega$, and $n=1,2, \ldots$ It is easy to see that each function $f_{n}$, 
$n=1,2, \ldots$ is $\partial-M_{t}^{K}-M_{t}^{D}$-integrable by appealing to Lemma 1 , and

$$
M_{t}^{z}\left(f_{n}\right)=\left[\mathrm{e}^{\mathrm{i} V t / n} \mathrm{e}^{-\mathrm{i} H_{0} t / z n}\right]^{n}, \quad n=1,2, \ldots
$$

For $z \in K$, the Riemann sums converge everywhere on $\Omega$, and by dominated convergence, $f_{n} \rightarrow \exp \left[-\mathrm{i} \int_{0}^{t} V \circ X_{s} d s\right]$ in $\mathrm{L}^{1}\left(M_{t}^{K}\right)$ as $n \rightarrow \infty$, and

$$
\lim _{n \rightarrow \infty} M_{t}^{z}\left(f_{n}\right)=\int_{\Omega} \exp \left[-\mathrm{i} \int_{0}^{t} V \circ X_{s} d s\right] d M_{t}^{z} .
$$

By virtue of Theorem 1, the left hand side converges uniformly for $z$ in compact subsets of $D$.

Now fix $\left.\left.J=\left\{t_{1}, \ldots, t_{j}\right\} \subset\right] 0, t\right]$ and let $B_{1}, \ldots, B_{j}$ be Borel subsets of $\boldsymbol{R}^{d}$. We suppose $0<t_{1}<\ldots<t_{j}<t$ and that $n$ is so large that $t / n<\min \left\{t_{1}, t_{2}-t_{1}, \ldots, t_{j}-t_{j-1}, t-t_{j}\right\}$.

Let $n_{k} t / n<t_{k} \leq\left(n_{k}+1\right) t / n, k=1,2, \ldots, j$. Then

$$
\begin{aligned}
& f_{n} M_{t}^{z}\left\{X_{t_{1}} \in B_{1}, \ldots, X_{t_{j}} \in B_{j}\right\}=\mathrm{e}^{-\mathrm{i} V t / n} S^{z}(t / n) \ldots \mathrm{e}^{-\mathrm{i} V t / n} S^{z}(t / n) \mathrm{e}^{-\mathrm{i} V t / n} \\
& S^{z}\left(\left(n_{j}+1\right) t / n-t_{j}\right) B_{j} S^{z}\left(t_{j}-n_{j} t / n\right) e^{-\mathrm{i} V t / n} S^{z}(t / n) \ldots e^{-\mathrm{i} V t / n} S^{z}(t / n) \mathrm{e}^{-\mathrm{i} V t / n} \\
& S^{z}\left(\left(n_{j-1}+1\right) t / n-t_{j-1}\right) B_{j-1} S^{z}\left(t_{j-1}-n_{j-1} t / n\right) \ldots \\
& \vdots \\
& S^{z}\left(t_{1}-n_{1} t / n\right) \mathrm{e}^{-\mathrm{i} V t / n} S^{z}(t / n) \ldots \mathrm{e}^{-\mathrm{i} V t / n} S^{z}(t / n)
\end{aligned}
$$

By [2] Lemma 4.3, it follows that $f_{n} M_{t}^{z}\left\{X_{t_{1}} \in B_{1}, \ldots, X_{t_{j}} \in B_{j}\right\}$, $n=1,2, \ldots$ converges in the strong operator topology to

$$
\begin{gathered}
\mathrm{e}^{-\mathrm{i}\left(H_{0} / z+V\right)\left(t-t_{j}\right)} B_{j} \mathrm{e}^{-\mathrm{i}\left(H_{0} / z+V\right)\left(t_{j}-t_{j-1}\right)} B_{j-1} \ldots \mathrm{e}^{-\mathrm{i}\left(H_{0} / z+V\right)\left(t_{2}-t_{1}\right)} B_{1} \\
\mathrm{e}^{-\mathrm{i}\left(H_{0} / z+V\right) t_{1}}
\end{gathered}
$$

uniformly for $B_{1}, \ldots, B_{j}$ and for $z$ in compact subsets of $D$. Therefore $f_{n}, n=1,2, \ldots$ converges in $\partial \mathrm{L}^{1}\left(M_{t}^{K}, M_{t}^{D}\right)$, and (1) holds.

For $V$ bounded, but not necessarily continuous, we can take a regularization $V_{n}, \mathrm{n}=1,2, \ldots$ of $V$ by smooth functions, such that $\left|V_{n}\right| \leq\|V\|_{\infty}, n=1,2, \ldots$ and $V_{n} \rightarrow V$ a. e. as $n \rightarrow \infty$.

Then $\mathrm{e}^{-\mathrm{i}\left(H_{0} / z+V_{n}\right) t} \rightarrow \mathrm{e}^{-\mathrm{i}\left(H_{0} / z+V\right) t}$ for each $z \in D$ and $t>0$ by strong resolvent convergence. To see that the convergence is uniform for $z$ in compact subsets of $D$, we apply Theorem 2 and the argument of [6] IX. 2. 4; the convergence of the second Neumann series is uniform for $z$ in compact subsets of $D$, and for each perturbation $V_{n}, n=1, \ldots, \mathrm{V}$. Another application of [2] Lemma 4.3 yields the convergence of $\exp \left(-\mathrm{i} \int_{0}^{t} V_{n} \circ X_{s} d s\right), n=1,2, \ldots$ to $\exp \left(-\mathrm{i} \int_{0}^{t} V \circ X_{s} d s\right)$ in $\partial \mathrm{L}^{1}\left(M_{t}^{K}, M_{t}^{D}\right)$ 
and (1).

Now truncate the positive and negative parts of $V$ to obtain the bounded functions $V_{n, m}, n, m=1,2, \ldots$ The same argument is enough to establish the convergence in $\partial \mathrm{L}^{1}\left(M_{t}^{K}, M_{t}^{D}\right)$ of

$$
\exp \left(-\mathrm{i} \int_{0}^{t} V_{n, m} \circ X_{s} d s\right) \in \partial \mathcal{L}^{1}\left(M_{t}^{K}, M_{t}^{D}\right)
$$

as $n \rightarrow \infty$ and then $m \rightarrow \infty$; namely, [6] IX,2.4 and the relative boundedness of $V$ with respect to $H_{0}$, Theorem 2, and [2] Lemma 4.3. It then follows that the Feynman representation (1) is valid.

Remark. The corresponding result for complex potentials was proved in [2] 4.8. It is not possible to control the convergence of the approximating sequences for complex potentials on the boundary of $D$, so it is unreasonable to expect convergence of the integrals in $\partial \mathrm{L}^{1}\left(M_{t}^{K}, M_{t}^{D}\right)$.

\section{Appendix: Integration with Respect to Closable Set Functions}

A semi-algebra of subsets of a set $\Omega$ is a semi-ring [1] containing the set $\Omega$. Let $E$ be a locally convex space with a fundamental system $\mathscr{P}$ of seminorms defining the topology of $E$.

The space ba $(\mathscr{E}, E)$ of bounded additive [1] set functions $m: \mathscr{E} \rightarrow E$ on the semi-algebra $\mathscr{E}$ is equipped with the semivariation topology; that is, for any seminorm $p \in \mathscr{P}, p_{\mathscr{E}}:$ ba $(\mathscr{E}, E) \rightarrow[0, \infty[$ is defined by $p_{\mathscr{E}}(m)=\sup p(m(\mathscr{E}))$ for each $m \in$ ba $(\mathscr{E}, E)$-the collection $\left\{p_{\mathscr{E}}: p \in \mathscr{P}\right\}$ then defines the semivariation topology on ba $(\mathscr{E}, E)$.

Let $Z$ be a directed set and $\left\langle\mathscr{S}_{\zeta}\right\rangle_{\zeta \in Z}$ an increasing family of semi-algebras. Set $\mathscr{S}=\bigcup_{\zeta \in Z} \mathscr{S}_{\zeta}$ and let $\mathrm{ba}\left(\mathscr{S}_{\zeta}, E\right)$ be the projective limit of the spaces ba $\left(\mathscr{S}_{\zeta}, E\right), \zeta \in Z$ linked by the restriction maps. Then ba $\left(\mathscr{S}_{\zeta}, E\right)$ is naturally identified with a space of additive set functions on the semi-algebra $\mathscr{S}$ which are locally bounded.

Let $W_{0}, W_{1}$ be index sets and let $\Gamma$ be a collection of families $\Gamma_{\xi}, \xi \in W_{0}$ of measures $\mu: \sigma(\mathscr{S}) \rightarrow[0, \infty[$ on the $\sigma$-algebra $\sigma(\mathscr{S})$ generated by $\mathscr{S}$ such that for each $\xi \in W_{0}, \sup \left\{\mu(\Omega): \mu \in \Gamma_{\xi}\right\}<\infty$ 。

Let $\Lambda$ be a collection of families $\Lambda_{\xi}, \xi \in W_{1}$ of $E$-valued additive set functions $\mu \in \mathrm{ba}\left(\mathscr{S}_{\xi}, E\right)$ such that for each $\xi \in W_{1}, \Lambda_{\xi}$ is a bounded 
subset of ba $\left(\mathscr{S}_{\zeta}, E\right)$.

The space of finite linear combinations of characteristic functions of sets belonging to $\mathscr{S}$ is denoted by $\operatorname{sim}(\mathscr{S})$. If $s \in \operatorname{sim}(\mathscr{S})$ and $m \in \mathrm{ba}\left(\mathscr{S}_{\zeta}, E\right)$, then $s m: \mathscr{S} \rightarrow E$ is the indefinite integral of $s$ with respect to $m$, defined in the obvious way; clearly $s m \in$ ba $\left(\mathscr{S}_{\zeta}, E\right)$.

Two topologies $\tau_{\Gamma}$ and $\tau_{\Lambda}$ are defined on $\operatorname{sim}(\mathscr{S}) . \leftarrow$ The first, $\tau_{\Gamma \text {, }}$ is defined by the family of seminorms $s \mapsto \sup _{\mu \in \Gamma_{\xi}} \mu(|s|), s \in \operatorname{sim}(\mathscr{S})$ as $\xi$ ranges over $W_{0}$, and the second is coarsest such that for each $\xi \in W_{1}, s \mapsto s m, s \in \operatorname{sim}(\mathscr{S})$ is an equicontinuous family of linear maps from $\operatorname{sim}(\mathscr{S})$ to $\mathrm{ba}\left(\mathscr{S}_{\zeta}, E\right)$ as $m$ ranges over $\Lambda_{\xi}$.

The topologies $\tau_{\Gamma}, \tau_{\Lambda}$ may not be Hausdorff, so let $\operatorname{sim}_{\Gamma}(\mathscr{S})$, $\operatorname{sim}_{\Lambda}(\mathscr{S})$ be their respective quotient spaces. In addition, it is supposed that the identity map $I: \operatorname{sim}(\mathscr{S}) \rightarrow \operatorname{sim}(\mathscr{S})$ factors into a map $I_{\Gamma \Lambda}: \operatorname{sim}_{\Gamma}(\mathscr{S}) \rightarrow \operatorname{sim}_{\Lambda}(\mathscr{S})$.

Now let $\mathrm{L}^{1}(\Gamma)$ be the space of (equivalence classes of) $\Gamma$-integrable functions introduced by Kluvánek [7] page 40. If $\mathrm{L}^{1}(\Gamma)$ is complete, then $\Gamma$ is said to be a closed system of measures [2], and in this case, the completion $\overline{\operatorname{sim}}_{\Gamma}(\mathscr{S})$ of $\operatorname{sim}_{\Gamma}(\mathscr{S})$ may be identified with a closed subspace of $\mathrm{L}^{1}(\Gamma)$, which in practice is all of $\mathrm{L}^{1}(\Gamma)$ (for example, $\Gamma_{\xi}$ is uniformly countably additive for each $\xi \in W_{0}$ ).

If $\Gamma$ is closed and the map $I_{\Gamma \Lambda}: \operatorname{sim}_{\Gamma}(\mathscr{S}) \rightarrow \operatorname{sim}_{\Lambda}(\mathscr{S})$ is a closable linear map from $L^{1}(\Gamma)$ into the completion $\overline{\operatorname{sim}}_{\Lambda}(\mathscr{S})$ of $\operatorname{sim}_{\Lambda}(\mathscr{S})$, then $\Lambda$ is $\Gamma$-closable.

The integration map $s \mapsto s m, s \in \operatorname{sim}(\mathscr{S})$ is clearly continuous for $\tau_{\Lambda}$ into ba $\left(\mathscr{S}_{\zeta}, E\right)$, so a function $f: \Omega \rightarrow \mathbb{C}$ is called $\Gamma$ - $\Lambda$-integrable if $f$ belongs to the domain $\mathscr{D}\left(\bar{I}_{\Gamma \Lambda}\right)$ of the closure $\bar{I}_{\Gamma \Lambda}$ of $I_{\Gamma \Lambda}$, and the image of $f$ via the (continuous extension of) the integration map $\mathrm{m}$ belongs to ba $\left(\mathscr{S}_{\zeta}, E\right)$ for all $m \in \cup \Lambda$.

If $E$ is complete, then this last condition holds whenever $f \in \mathscr{D}\left(\bar{I}_{\Gamma \Lambda}\right)$. The uniquely defined image of $f$ by $\circ \mathrm{m}$ is denoted, of course, by $\mathrm{fm}$; it is the indefinite integral of $f$ with respect to $m$.

A convergence theorem for these indefinite integrals can be read straight off the closedness property of the map $\bar{I}_{\Gamma \Lambda}$ [2] Theorem 2.5.

To apply the definition to Schrödinger semigroups, set

$$
K=\{a \mathrm{i}: a>0\} ; D=\{z \in \mathbb{C}: \operatorname{Im} z \geq 0, \quad z \neq 0\}
$$




$$
S^{z}(t)=\mathrm{e}^{1 \Delta t / 2 z}
$$

for each $z \in D$ and $t>0$. The operator $\Delta$ is the self-adjoint extension of the Laplacian $\partial^{2} / \partial x_{1}^{2}+\ldots+\partial^{2} / \partial x_{d}^{2}$ on $\mathrm{L}^{2}\left(\boldsymbol{R}^{d}\right)$. The exponential is defined by the operational calculus for self-adjoint operators.

Let $\Omega$ be the set of all continuous functions $\omega:[0, \infty] \rightarrow \boldsymbol{R}^{d}$, and set $X_{t}(\omega)=\omega(t), \omega \in \Omega, t>0$. The Borel $\sigma$-algebra of $\boldsymbol{R}^{d}$ is denoted by $\Sigma$. A set will sometimes be identified with its characteristic function, and a Borel measurable function will also be identified with the operator on $\mathrm{L}^{2}\left(\boldsymbol{R}^{d}\right)$ it defines.

For each $t>0, z \in D$, define

$$
\begin{gathered}
M_{t}^{z}\left\{X_{t_{1}} \in B_{1}, \ldots, X_{t_{k}} \in B_{k}\right\}=S^{z}\left(t-t_{k}\right) B_{k} S^{z}\left(t_{k}-t_{k-1}\right) \ldots \\
B_{2} S^{z}\left(t_{2}-t_{1}\right) B_{1} S^{z}\left(t_{1}\right)
\end{gathered}
$$

for all $0<t_{1}<\ldots<t_{k} \leq t, B_{1}, \ldots, B_{k} \in \Sigma, k=1,2, \ldots$ Then $M_{t}^{z}: \mathscr{S}_{t} \rightarrow$ $\mathscr{L}\left(\mathrm{L}^{2}\left(\mathbb{R}^{d}\right)\right)$ is an operator-valued set function on the semi-algebra of sets of the form $\left\{X_{t_{1}} \in B_{1}, \ldots, X_{t_{k}} \in B_{k}\right\}, 0<t_{1}, \ldots, t_{k} \leq t, B_{1}, \ldots, B_{k} \in \Sigma$, $k=1,2, \ldots$.

For each $z \in K, M_{t}^{z}$ is the restriction to $\mathscr{S}_{t}$ of a unique $\mathscr{L}\left(\mathrm{L}^{2}\left(\boldsymbol{R}^{d}\right)\right)$ valued measure, also denoted by $M_{t}^{z}$, on $\sigma\left(\mathscr{S}_{t}\right)$. This follows by representing $M_{t}^{z}$ in terms of the Wiener process [2].

Our space $E$ will be the space $H_{c}(D)$ of continuous functions on $D$ which are analytic in the interior $D^{\circ}$ of $D$, equipped with the topology of uniform convergence on compact subsets of $D^{\circ}$ (it is not complete):

For each $\phi, \phi \in \mathrm{L}^{2}\left(\boldsymbol{R}^{d}\right),\left(M_{t}^{D} \phi, \phi\right)$ represents the $H_{c}(D)$-valued set function defined by

$$
\left(M_{t}^{D} \phi, \phi\right)(A)(z)=\left(M_{t}^{z}(A) \phi, \phi\right),
$$

$A \in \mathscr{S}_{t}, \phi, \phi \in \mathrm{L}^{2}\left(\boldsymbol{R}^{d}\right), z \in D$ and $t>0$.

Finally, for each $t>0$, our increasing family of semi-algebras is the family $\left\langle\mathscr{S}_{J}\right\rangle_{J \in \mathscr{T}_{t}}$ of semi-algebras $\mathscr{S}_{J}$ of sets of the form $\left\{X_{t_{1}} \in B_{1}, \ldots\right.$, $\left.\left.\left.X_{t_{j}} \in B_{j}\right\}, B_{1}, \ldots, B_{j} \in \Sigma, J=\left\{t_{1}, \ldots, t_{j}\right\} \subset\right] 0, t\right]$. The set $\mathscr{F}_{t}$ is the collection of all finite sets $J \subset] 0, t]$ directed by inclusion.

Put $\Gamma_{t}^{a, \phi}=\left\{\left|\left(M_{t}^{a \mathrm{i}} \phi, \phi\right)\right|: \phi \in \mathrm{L}^{2}\left(\boldsymbol{R}^{d}\right),\|\psi\|_{12} \leq 1\right\}$ and $\Gamma_{t}=\left\{\Gamma_{t}^{a, \phi}: a>0\right.$, $\left.\phi \in \mathrm{L}^{2}\left(\boldsymbol{R}^{d}\right),\|\phi\|_{2} \leq 1\right\}$ for each $t>0$. Here $|\cdot|$ denotes the variation (measure) of a complex measure on the $\sigma$-algebra $\sigma\left(\mathscr{S}_{t}\right)$.

For each $t>0, \Lambda_{t}^{\phi}=\left\{\left(M_{t}^{D} \phi, \phi\right): \phi \in \mathrm{L}^{2}\left(\boldsymbol{R}^{d}\right),\|\psi\|_{2} \leq 1\right\}$, and 
$\Lambda_{t}=\left\{\Lambda_{t}^{\phi}: \phi \in \mathrm{L}^{2}\left(\boldsymbol{R}^{d}\right),\|\phi\|_{2} \leq 1\right\}$.

A $\Gamma_{t}-\Lambda_{t}$-integrable function is said to be $M_{t}^{K}-M_{t}^{D}$-integrable. A $\Gamma_{t}$-integrable function [7] III. 1 is said to be $M_{t}^{K}$-integrable. For each $M_{t}^{K}-M_{t}^{D}$-integrable function $f$, the additive operator-valued set functions

$$
f M_{t}^{z}: \mathscr{S}_{t} \rightarrow \mathscr{L}\left(\mathrm{L}^{2}\left(\boldsymbol{R}^{d}\right)\right), \quad z \in D
$$

can be read off from the definitions in the obvious way [2].

These are our integrals. For $z \in K$, they correspond to the usual integrals with respect to an operator-valued measure [7], and they are analytic continuations of these off $K$; that is, for each $t>0$ and $A \in \mathscr{S}_{t}, f M_{t}(A)$ is continuous for the weak operator topology on all of $D$, and analytic in the interior of $D$.

The space $\mathrm{L}^{1}\left(\Gamma_{t}\right)$ is written as $\mathrm{L}^{1}\left(M_{t}^{K}\right)$ for each $t>0$. The space $\mathrm{L}^{1}\left(M_{t}^{K}, M_{t}^{D}\right)$ of all (equivalence classes of) $M_{t}^{K}-M_{t}^{D}$-integrable functions is equipped with the coarsest topology for which both the inclusion of $\mathrm{L}^{1}\left(M_{t}^{K}, M_{t}^{D}\right)$ in $\mathrm{L}^{1}\left(M_{t}^{K}\right)$ and the map $\bar{I}_{\Gamma_{t} \Lambda_{t}}$ are continuous. Unfortunately, $\mathrm{L}^{1}\left(M_{t}^{K}, M_{t}^{D}\right)$ is not complete because $H_{c}(D)$ is not complete; we shall learn to live with this fact.

Expressions such as " $M_{t}^{K}$-a. e.", " $M_{t}^{K}$-null" have the obvious meanings attached to them in [2]. The set function $M_{t}^{1}$ is written as $M_{t}^{F}, t>0$.

\section{References}

[1] Halmos, P., Measure Theory, Van Nostrand, New York, 1950.

[2] Jefferies, B., Integration with respect to closable set functions, J. Funct. Anal., in press. $[3]$ submitted.

[4] The generation of weakly integrable semigroups, J. Funct. Anal., in press.

[5] Johnson, G. W. and Skoug, D. L., Feynman integrals of non-factorable finite-dimensional functionals, Pacific. J. Math. 45 (1973), 257-274.

[6] Kato, T., Perturbation Theory for Linear Operators, Springer-Verlag, Berlin, Heidelberg, New York, 1980.

[7] Kluvánek, I. and Knowles, G., Vector Measures and Control Systems, North-Holland, Amsterdam, 1976.

[8] Nelson, E., Feynman integrals and the Schrodinger equation, J. Math. Phys. 5 (1964), 332-343.

[9] Trotter, H. F., Approximation of semi-groups of operators, Pacific J. Math. 8 (1958), 887-919.

[10] On the product of semi-groups of operators, Proc. Amer. Math. Soc. 10 (1959), 545-551. 
